INPLASY

PROTOCOL

To cite: Hou et al.

Effectiveness and safety of Wendan Decoction for poststroke depression: A protocol for systematic review and meta-analysis. Inplasy protocol 2021110018. doi:

10.37766/inplasy2021.11.0018

Received: 06 November 2021

Published: 06 November 2021

Corresponding author:

Yonghui Hou

houyonghui11@163.com

Author Affiliation:

Shijiazhuang People's

Hospital.

Support: National Natural Science Found.

Review Stage at time of this submission: The review has not yet started.

Conflicts of interest:

None declared.

\section{Effectiveness and safety of Wendan Decoction for post-stroke depression: A protocol for systematic review and meta-analysis}

Hou, Y1; Gao, J2; Pang, W3; Si, W4.

Review question / Objective: To evaluate the effectiveness and safety of Wendan Decoction in the treatment of poststroke depression disease.

Information sources: PubMed, Cochrane Library, Embase, China National Knowledge Infrastructure(CNKI), Wanfang Database, China Bioloy Medicine(CBM) and VIP Data base will be searched from inception to November 2021.

Main outcome(s): The primary outcomes will include the Hamilton depression scale score and the effective rate. As previously reported, a $\geq 25 \%$ reduction in the Hamilton depression scale score was indicative of effective treatment.

INPLASY registration number: This protocol was registered with the International Platform of Registered Systematic Review and Meta-Analysis Protocols (INPLASY) on 06 November 2021 and was last updated on 06 November 2021 (registration number INPLASY2021110018).

\section{INTRODUCTION}

Review question / Objective: To evaluate the effectiveness and safety of Wendan Decoction in the treatment of post-stroke depression disease.

Condition being studied: Post-stroke depression is a common stroke complication characterized by hopelessness, anxiety, disordered sleep, and lowered responsiveness. In the initial 2 post-stroke months, about $36 \%$ of the patients develop signs of depression with $14 \%$ being diagnosed with major depressive disorder. In the People's Republic of China, 7.5 million stroke survivors have been reported; moreover, 
there are 3 million patients with post-stroke depression. Post-stroke depression has harmful motor and cognitive effects and negatively affects functional recovery. The first choice antidepressant treatments for patients with a post-stroke depression diagnosis are well established. However, antidepressants are associated with several adverse side effects, including mouth dryness, fatigue, drowsiness, weight gain, and sexual dysfunction. This, in turn, leads to low patient compliance rates. These negative factors may force poststroke depression patients or clinicians to explore other treatment options. Therefore, it is crucial to provide better treatment strategies for post-stroke depression patients. Many studies have shown that Chinese medicine, acupuncture and other methods have achieved good results in the treatment of hyperlipidemia. Among which, Wendan Decoction is a prescription worthy of attention. However, no systematic studies have been found on the efficacy and safety of Wendan Decoction in the treatment of post-stroke depression disease. So this research aims to systematically and comprehensively evaluate the effectiveness and safety of Wendan Decoction in the treatment of post-stroke depression.

\section{METHODS}

Search strategy: The key search terms are "Wendan Decoction" AND "post-stroke depression".

Participant or population: Patients diagnosed with post-stroke depression will be participants without restrictions of age, gender, race, or duration of disease.

\section{Intervention: Wendan Decoction.}

Comparator: In the control group, the intervention means may include medicine(western medicine), routine symptomatic treatment, etc.

Study designs to be included: Only randomized controlled trials(RCTs) will be included in this study.
Eligibility criteria: Published or unpublished randomized controlled trials with Chinese or English.

Information sources: PubMed, Cochrane Library, Embase, China National Knowledge Infrastructure(CNKI), Wanfang Database, China Bioloy Medicine(CBM) and VIP Data base will be searched from inception to November 2021.

Main outcome(s): The primary outcomes will include the Hamilton depression scale score and the effective rate. As previously reported, $a \geq 25 \%$ reduction in the Hamilton depression scale score was indicative of effective treatment.

Additional outcome(s): The secondary outcomes were the National Institute of Health Stroke Scale scores, Barthel index, and the incidence of adverse events.

Quality assessment / Risk of bias analysis: The Cochrane Handbook for systematic reviews of interventions Version 6 will be performed to assess a broad category of biases in the included studies. We will evaluate biases from the following seven aspects: random sequence generation, allocation concealment, blinding of the participants and personnel, blinding of the outcome assessments, incomplete outcome data, selective reporting, and other sources of bias. These studies will be assigned as low Risk, high Risk or unclear Risk. Inconsistencies will be resolved by discussion with other reviewers.

Strategy of data synthesis: If studies are adequately homogeneous in design and comparison, we will conduct data synthesis using Review Manager Software 5.4. The fixed-effects or random-effects model will be chosen depending on the 12 value. A $95 \%$ confidence interval will be the effective size for data synthesis. We will perform qualitative analysis if the data is not fit for quantitative analysis.

Subgroup analysis: In the case of high heterogeneity, subgroup analysis will be done to identify the sources of heterogeneity. We will conduct subgroup 
analysis according to different combinations of moxibustion and other combined therapies, different course time, or different outcome indicators.

Sensitivity analysis: When there are adequate studies, sensitivity analysis will be adopted for primary outcomes to explore the robustness of conclusions if feasible and assess the impact of method quality, sample size, and missing data. The meta-analysis will be conducted again after excluding the lower quality research. The results will be compared and discussed according to the results.

Country(ies) involved: China.

Keywords: post-stroke depression; wendan decoction; meta-analysis; systematic review.

Contributions of each author:

Author 1 - Yonghui Hou.

Author 2 - Jing Gao.

Author 3 - Wenwen Pang.

Author 4 - Wei Si. 